Biomédica de Málaga. Hospital Regional Universitario de Málaga, Reumatología, Málaga, Spain

Objectives: To evaluate the worldwide incidence and prevalence of ANCA vasculitis through a systematic review of the literature and meta-analysis.

Methods: A systematic search of MEDLINE and EMBASE search engines was carried out for studies that analyzed the incidence and prevalence of ANCA vasculitis in different geographical areas. Inclusion criteria: patients diagnosed with ANCA vasculitis according to ACR criteria/ Chapel Hill Consensus and adult patients (> 16 years). All ANCA vasculitis (microscopic polyangiitis, granulomatosis with polyangiitis, eosinophilic granulomatosis with polyangiitis) were considered. Exclusion criteria: editorials, conference abstracts, case or cases series reports and narrative reviews; insufficient description of the methods; lack of data to compute incidence or prevalence; and duplicate studies.Variables: Main variable: the pooled prevalence measured by the number of prevalent cases per million / person-year $(95 \% \mathrm{Cl})$ and the pooled incidence measured as the number of incident cases per million / person-year $(95 \% \mathrm{Cl})$. Secondary variables: the prevalence and incidence of each vasculitis ANCA and according geographic area. A meta-analysis was undertaken to estimate the pooled incidence and the pooled prevalence per million / person-years. The $95 \% \mathrm{Cl}$ and $\mathrm{I} 2$ for heterogeneity were calculated. Results: Twenty four studies were included. The pooled incidence $(95 \% \mathrm{Cl})$ was 12.2 per million / person-year (8.4-16.5) and the pooled prevalence $(95 \%$ $\mathrm{Cl})$ was 130 per million / person-year (67.5-213). The individual incidence for each vasculitis was: GPA (6.7), MPA (5.9) and EGPA (1.6). The individual prevalence for each vasculitis was: GPA (69.3), MPA (21.9) and EGPA (13.5).

In the analysis by continents, the pooled incidence for GPA vasculitis was higher in Europe (7.5), while the pooled incidence for MPA vasculitis was higher in America (6.9) and for EGPA vasculitis it was higher in Asia (1.8). The pooled prevalence for GPA and MPA vasculitis was higher in Europe (83.9,24.4 respectively) than in America (14.2, 12.8 respectively).

Conclusion: The pooled incidence and the pooled prevalence are higher in the case of GPA vasculitis compared to the rest of ANCA vasculitis. In general there is a predominance of incidence and prevalence of all ANCA vasculitis in the northern hemisphere compared to the south.

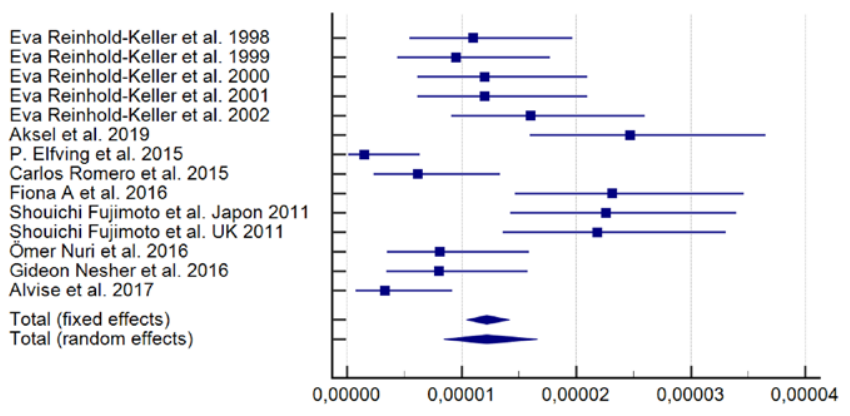

Figure 1. The pooled incidence ANCA vasculitis.

Fure 1. The pooled incidence ANCA vasculitis.

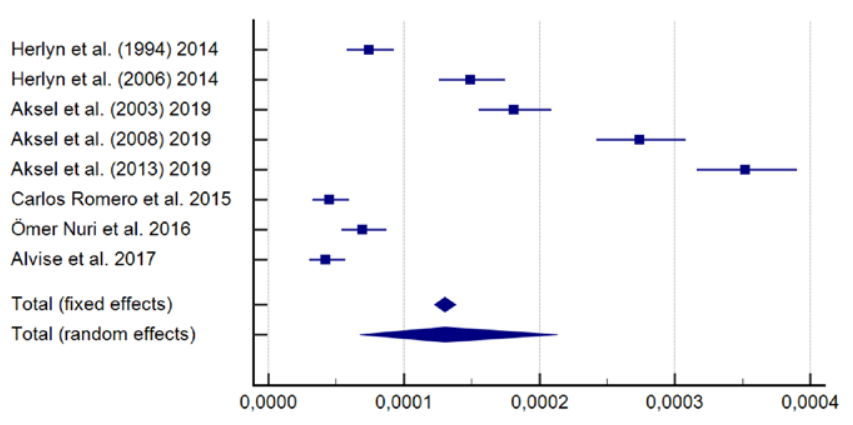

Figure 2. The pooled prevalence ANCA vasculitis

Disclosure of Interests: None declared

DOI: 10.1136/annrheumdis-2021-eular.1630

\section{POS0247 \\ INTERSTITIAL ANCA-ASSOCIATED VASCULITIS ASSOCIATES WITH SEVERE KIDNEY INJURY INDEPENDENT OF GLOMERULONEPHRITIS}

S. Hakroush ${ }^{1}$, B. Tampe ${ }^{2}{ }^{1}$ University Medical Center Göttingen, Institute of Pathology, Göttingen, Germany; ${ }^{2}$ University Medical Center Göttingen, Department of Nephrology and Rheumatology, Göttingen, Germany
Background: Antineutrophil cytoplasmic antibody (ANCA)-associated vasculitis (AAV) is a small vessel vasculitis affecting multiple organ systems, including the kidney. Small vessels in the kidney include small-sized arteries (interlobular artery, afferent and efferent arteriole), capillaries (glomerular and peritubular capillary) and venules.

Objectives: Although crescentic ANCA glomerulonephritis (GN) is a common histological finding reflecting glomerular small vessel vasculitis, it is reasonable that manifestation of AAV could also contribute to interstitial small vessel vasculitis. Therefore, we here aimed to expand our current knowledge focusing on interstitial vasculitis in ANCA GN by systematic histological scoring of vascular lesions analogous to Banff.

Methods: A total number of 49 kidney biopsies with confirmed renal involvement of AAV at the University Medical Center Göttingen were retrospectively included between 2015 till 2020. A renal pathologist (SH) evaluated all biopsies and was blinded to clinical data collection and analysis. A detailed methological section is provided in the Supplementary material and methods section.

Results: Since previous studies established that crescentic ANCA GN associates with severe kidney injury and acute deterioration of kidney function in AAV, we first systematically scored interstitial vasculitis in association with requirement of renal replacement therapy (RRT). Among all active and chronic tubulointerstitial lesions analogous to the Banff scoring system, the only association between severe kidney injury requiring RRT was observed for interstitial vasculitis in AAV reflected by peritubular capillaritis ( $p t c, p=0.0002)$ and arteritis $(v, p=0.0069)$, affecting $5 / 49(10.2 \%)$ and $11 / 49(22.4 \%)$ of renal biopsies, respectively. Since it is known that severe deterioration of kidney function also correlates with crescentic ANCA GN, we next directly compared glomerular and tubulointerstitial lesions. The fraction of normal glomeruli was inversely associated with interstitial fibrosis (ci), total (ti) and inflammation in IFTA (i-IFTA), whereas glomerular crescents were associated with interstitial inflammation (i), tubulitis (t) and total inflammation (ti). In contrast, global glomerular sclerosis associated with less interstitial inflammation (i) but correlated with interstitial fibrosis (ci) and tubular atrophy (ct), confirming established mechansim that chronic glomerular injury leads to tubular atrophy and interstitial fibrosis. Interestingly, no association between interstitial vasculitis (ptc and v correlating with severe kidney injury) and any glomerular lesion in ANCA GN (also correlating with severe kidney injury) was observed, thereby confirming that interstitial vasculitis contributes to severe kidney injury independent of ANCA GN. By contrast, short-term renal recovery from RRT was equal in both groups, suggesting a distinct association with acute decline of kidney function at disease onset.

Conclusion: Taken together, by using the Banff scoring system we here expand our current knowledge of renal interstitial lesions in AAV revealing peritubular capillaritis and arteritis as important histological alterations associated with severe kidney injury in a considerable subset of AAV. Furthermore, our findings that interstitial vasculitis did not correlate with crescentic ANCA GN implicate that the characteristics of each vasculitis manifestation are independent and could further improve our understanding of mechanisms contributing to renal injury. These observations suggest that interstitial vasculitis in AAV may also affect long-term prognosis requiring further investigation.

Disclosure of Interests: None declared

DOI: 10.1136/annrheumdis-2021-eular.4296

\begin{tabular}{|l|}
\hline POS0248 \\
PSYCHOMETRIC PROPERTIES OF OUTCOME \\
MEASUREMENT INSTRUMENTS FOR ANCA- \\
ASSOCIATED VASCULITIS: A SYSTEMATIC \\
LITERATURE REVIEW
\end{tabular}

A. Berti ${ }^{1,2}$, G. Boleto ${ }^{3}$, P. A. Merkel ${ }^{4}$, G. Tomasson ${ }^{5}$, S. Monti ${ }^{6}$, K. A. Quinn ${ }^{7}$, L. Carmona ${ }^{8}$, S. Ramiro ${ }^{9,10}$ on behalf of OMERACT Vasculitis Working Group. ${ }^{1}$ Hospital of Trento, University of Trento, Rheumatology, Trento, Italy; ${ }^{2}$ Mayo Clinic, Thoracic Disease Research Unit, Rochester, United States of America; ${ }^{3}$ Cochin Hospital, Rheumatology, Paris, France; ${ }^{4}$ Penn Medicine, University of Pennsylvania Health System, Rheumatology, Philadelphia, United States of America; ${ }^{5}$ Landspitali The National University Hospital of Iceland, Rheumatology, Reykjavik, Iceland; ${ }^{6}$ University of Pavia, Rheumatology, Pavia, Italy; ${ }^{7}$ National institute of arthritis and musculoskeletal and skin diseases, Rheumatology, Bethesda, United States of America: ${ }^{8}$ Instituto de Salud Musculoesquelética (INMUSC), Rheumatology, Madrid, Spain; ${ }^{9}$ Department of Rheumatology, Leiden University Medical Center, Rheumatology, Leiden, Netherlands; ${ }^{10}$ Department of Rheumatology, Zuyderland Medical Center, Rheumatology, Heerlen, Netherlands

Background: The OMERACT Vasculitis Working Group has defined a Core Domain Set of outcome measures for ANCA-associated vasculitis (AAV). However, the psychometric properties of available outcome measurement instruments in AAV, an essential consideration when choosing among instruments, have not been summarized.

Objectives: To systematically review and summarize the psychometric properties of outcome measurement instruments used in AAV. 
Methods: A comprehensive search of several databases (Medline, EMBASE, Cochrane, Scopus, among others) from inception to July 14, 2020 and without language limitations was conducted. Articles were included if they covered psychometric properties of instruments used in AAV (granulomatosis with polyangiitis, GPA; microscopic polyangiitis, MPA; eosinophilic granulomatosis with polyangiitis, EGPA); articles encompassing other systemic vasculitides and not presenting the data for AAV separately were excluded. Following the COSMIN and OMERACT frameworks, different psychometric properties (validity, inter- and intra-observer reliability, sensitivity to change, and feasibility) of outcome measurement instruments used in AAV were assessed. Risk of bias was assessed according to the COSMIN checklist.

Results: From 2505 articles identified, 20 met the predefined criteria. Three were identified as development studies, 14 were validation studies, and 3 pursued both objectives.

These studies provided information on 16 instruments: 8 assessing disease activity, 1 assessing disease damage, 3 assessing patient-reported outcome, 4 assessing function (Figure 1). Overall, a few psychometric properties have been considered in each study, ranging from one to five. Most of the instruments were tested in GPA only $(n=7)$, followed by AAV as a group (GPA, MPA and EGPA; $n=5)$, MPA and GPA $(n=3)$, and EGPA only $(n=1)$. Sample sizes of the studies ranged between 27 and 626 patients. The studies with a higher risk of bias, according to COSMIN definitions, were those assessing RAPID3, MVIA, ENT/ GPA DAS, and ODSS.

There was a wide heterogeneity of the psychometric proprieties assessed for each instrument. Validity was the most frequently assessed domain in $88 \%$ of the instruments, and few properties other than construct validity were reported (Figure 1).

Within each domain, BVAS/WG for activity, VDI for damage, AAV-PRO for patient-reported outcomes, and ODSS for function were the instruments with more psychometric features assessed. For the disease activity domain, BVAS/ WG showed a good validity having the highest correlation with physician global assessment $(r=0.90)$, a good reliability (intra-observer ICC $=0.62$; inter-observer $I C C=0.97$ ), and good feasibility and responsiveness. For disease damage, VDI showed moderate validity (correlations with BVAS/WG at 5-year with $r=0.20$ and BVAS/WG at 1-year with $r=0.40$ ) and good feasibility. Among patient-reported outcomes, AAV-PRO had the best performance in terms of validity (construct validity: correlations of the 6 disease domains and EQ-5D-5L, with $r$ ranging between -0.78 and -0.55 ; discriminating validity between active disease versus remission of the 6 disease domains, $p<0.0001$ for all comparisons). The performance of instruments assessing function domain was low-to-moderate.

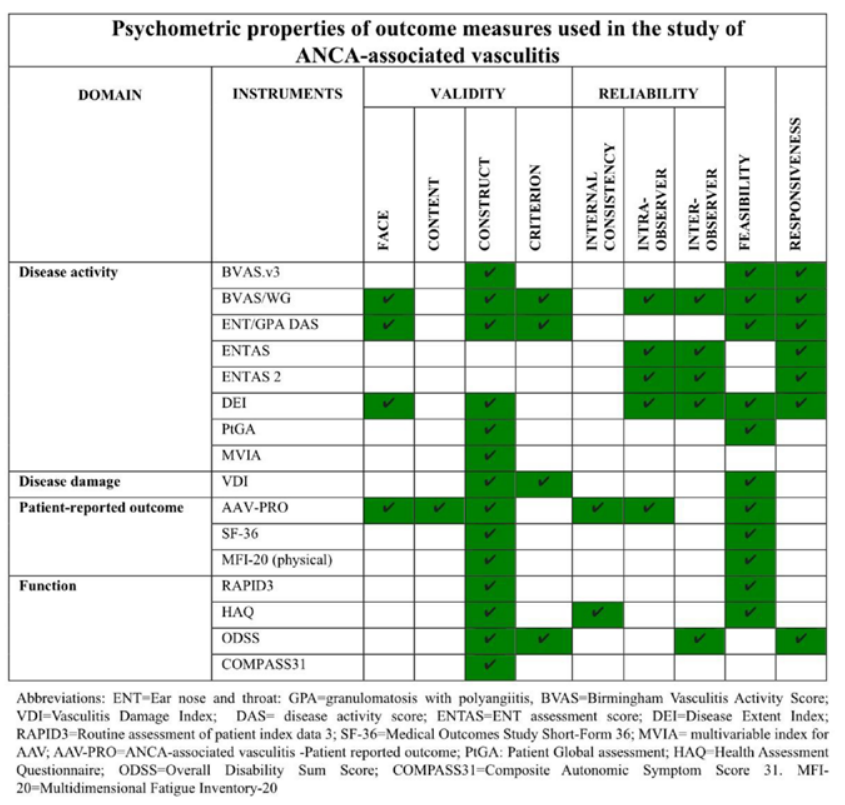

* Only the physical fatigue domain of the score was assessed (not the overall score)

Conclusion: Sixteen instruments covering the OMERACT domains of disease activity, damage, patient-reported outcome, and function had their psychometric properties assessed in the study of AAV. The majority were developed or validated for GPA only or AAV as a group. Overall, validity was the domain most frequently assessed. BVAS/WG, VDI, AAV-PRO, and ODSS were the instruments with more psychometric features assessed. More rigorous studies aimed at estimating a wider range of psychometric properties in larger numbers of patients with AAV are warranted.

\section{REFERENCES:}

[1] Castrejon I, et al. Clin Exp Rheumatol. 2015

[2] Merkel PA, Journal of Rheumatology, July 2011

Disclosure of Interests: None declared

DOI: 10.1136/annrheumdis-2021-eular.1451

\section{POS0249 THE EFFECT OF NASAL STAPHYLOCOCCUS AUREUS COLONIZATION ON DISEASE ACTIVITY AND THE EFFECT OF ANTIBIOTIC TREATMENT IN PATIENTS WITH ANCA ASSOCIATED VASCULITIS; A RETROSPECTIVE COHORT STUDY}

C. Schaap ${ }^{1}$, R. Krol ${ }^{1,2}$, H. H. F. Remmelts ${ }^{2}$, R. Klaasen ${ }^{3}$, E. Hagen ${ }^{2}$,

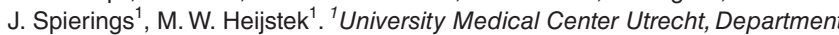
of Rheumatology and Clinical Immunology, Utrecht, Netherlands; ${ }^{2}$ Meander Medical Center, Department of Internal Medicine, Amersfoort, Netherlands; ${ }^{3}$ Meander Medical Center, Department of Rheumatology, Amersfoort, Netherlands

Background: Antineutrophil cytoplasmic antibodies (ANCA)-associated vasculitis (AAV) is a necrotizing vasculitis, predominantly affecting small or medium vessels with few or no immune deposits. Ear, nose and throat (ENT) involvement in $\mathrm{AAV}$ is frequently present. AAV has a relapsing-remitting disease course. A factor that has been associated with induction of relapses is nasal Staphylococcus aureus (S. aureus) colonization. This alleged association between nasal colonization and induction of relapses has resulted in the use of antibiotics. However the effect of antibiotic treatment on disease activity remains controversial. Some studies showed a beneficial effect of antibiotic treatment whereas other studies found no effect of antibiotic treatment on disease activity.

Objectives: The aim of this study was to identify the role of nasal S. aureus colonization and the effect of systemic or local antibiotic treatment on disease activity in patients with AAV

Methods: Clinical, laboratory and histological data from all AAV patients with ENT involvement, diagnosed in two medical centers between 1981 and 2020, were retrospectively collected. Nasal S. aureus colonization was defined as at least one positive nasal swab during follow-up. Data on systemic (cotrimoxazole and azithromycin) and local antibiotics (mupirocin) use was collected. Disease activity was divided into systemic and local disease activity. Systemic disease activity consisted of history of relapses, relapse number per patient years and Birmingham vasculitis activity score version 3 (BVAS3) at last visit. Local disease activity included history of ENT relapses, development of saddle nose deformity or subglottic stenosis during follow-up.

Results: Two-hundred and thirteen patients were included in the analysis. Median follow-up time was 8 (IQR 3 -17) years. S. aureus colonization was tested in 100 $(46.9 \%)$ cases of which 44 patients tested positive. Only one patient developed a subglottic stenosis, and 13 patients developed a saddle nose deformity during follow-up. Systemic and local disease activity at baseline and at last visit were comparable between patients with and without and S. aureus colonization. Regression analysis showed no difference in relapse number per patient year between AAV patients colonized with S. aureus versus non-colonized patients (RR $2.03 ; 95 \% \mathrm{Cl}$, $0.97-4.26, p=0.06$ ). This also accounted for local ENT relapses (OR 0.134 $95 \% \mathrm{Cl} 0.06-1.47, \mathrm{p}=0.14$ ) and saddle nose deformity (OR $0.61 ; 95 \% \mathrm{Cl} 0.04$ $10.68, p=0.74)$. Twenty-eight (13.1\%) $\mathrm{S}$. aureus positive patients received antibiotics aimed at eradication of S. aureus. Twenty-two (10.3\%) received cotrimoxazole, $2(0.9 \%)$ received azithromycin and $17(8.0 \%)$ patients received mupirocin ointment. No significant difference was found between the treated versus non-treated group with regard to systemic and local disease activity.

Conclusion: Nasal S. aureus colonization does not influence systemic or local disease activity. Antibiotic eradication treatment did not modify disease activity in this study.

Table 1. Effect of AB treatment on disease activity of $40 \mathrm{AAV}$ patients with ENT involvement and $S$. aureus colonization

\begin{tabular}{|c|c|c|c|}
\hline \multirow[t]{2}{*}{ Disease activity } & \multicolumn{2}{|c|}{ Antibiotic treatment } & \multirow[b]{2}{*}{ P-value } \\
\hline & Yes $(n=28)$ & No $(n=12)$ & \\
\hline \multicolumn{4}{|l|}{ Systemic symptoms } \\
\hline History of one or more relapses, $\mathrm{n}(\%)$ & 18 (47.4\%) & $4(10.5 \%)$ & 0.635 \\
\hline $\begin{array}{l}\text { Relapse number per patient years, median } \\
\text { (IQR) * }\end{array}$ & $0.11(0-0.18)$ & $\begin{array}{l}0.17(0.02 \\
-0.26)\end{array}$ & 0.346 \\
\hline $\begin{array}{l}\text { BVAS3 last visit, median (IQR) } \\
\text { Local symptoms }\end{array}$ & $1(0-4)$ & $1(0-4)$ & 0.932 \\
\hline History of one or more ENT relapses, $\mathrm{n}(\%)$ * & $9(33.3 \%)$ & $3(11.1 \%)$ & 0.438 \\
\hline $\begin{array}{l}\text { Development of saddle nose deformity during } \\
\text { follow-up, } \mathrm{n}(\%)^{*}\end{array}$ & $4(12.1 \%)$ & $0(0 \%)$ & 0.367 \\
\hline
\end{tabular}

Values are median (interquartile range IQR) or $n$ (\%). BVAS3: Birmingham Vasculitis Activity Score version 3; ANCA: Anti-Neutrophilic Cytoplasmic Autoantibody; AAV: ANCA associated vasculitis; ENT: ear nose and throat * More than $10 \%$ missings in analysis. For an overview of the number of included patients per analysis, see supplementary table $B$. 